\title{
Isoflavonas de soya y salud humana: cáncer de mama y sincronización de la pubertad
}

\author{
LUIS VALLADARES ${ }^{\mathrm{a}}$, ARGELIA GARRIDO ${ }^{\mathrm{b}}$, WALTER SIERRALTA ${ }^{\mathrm{a}}$
}

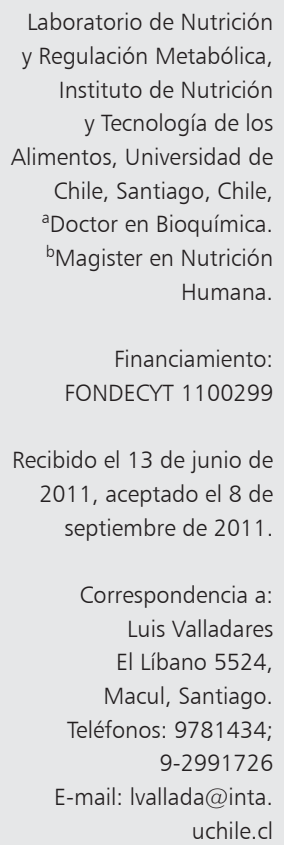

\section{Soy isoflavones and human health: breast cancer and puberty timing}

Accumulated exposure to high levels of estrogen is associated with an increased incidence of breast cancer. Thus, factors such as early puberty, late menopause and hormone replacement therapy are considered to be risk factors, whereas early childbirth, breastfeeding and puberty at a later age are known to consistently decrease the lifetime breast cancer risk. Epidemiological studies suggest that consumption of isoflavones correlates with a lower incidence of breast cancer. Data from human intervention studies show that the effects of isoflavones on early breast cancer markers differ between pre- and post-menopausal women. The reports from experimental animals (rats and mice) on mammary tumors are variable. These results taken together with heterogeneous outcomes of human interventions, have led to a controversy surrounding the intake of isoflavones to reduce breast cancer risk. This review summarizes recent studies and analyzes factors that could explain the variability of results. In mammary tissue, from the cellular endocrine viewpoint, we analyze the effect of isoflavones on the estrogen receptor and their capacity to act as agonists or antagonists. On the issue of puberty timing, we analyze the mechanisms by which girls, but not boys, with higher prepuberal isoflavone intakes appear to enter puberty at a later age.

(Rev Med Chile 2012; 140: 512-516).

Key words: Breast neoplasms; Isoflavones; Phytoestrogens; Puberty.
U n notable aumento en el consumo de alimentos derivados del poroto de soya, Glycine max, ha ocurrido en los últimos años como consecuencia de la creencia que su consumo produce beneficios en la salud, independientemente del contenido de sus nutrientes. $\mathrm{Al}$ igual que todos los alimentos, el poroto de soya contiene además diversos compuestos biológicamente activos, investigados ampliamente y que incluyen, entre otras, saponinas, lunasina e isoflavonas (ISO), estas últimas responsables del gran interés científico por esta leguminosa ${ }^{1}$.

En los alimentos de consumo humano tradicional las ISO tienen una distribución limitada y sólo en el poroto de soya se encuentran en cantidades fisiológicamente relevantes. Las principales
ISO presentes en semillas de la leguminosa son la genistina, la daidzina y la glicitina, conjugados glicosilados de genisteina, daidzeina y gliciteina, respectivamente; que son las moléculas activas para los humanos. Las ISO glicosiladas no se pueden absorber como tal, pero en el intestino son hidrolizadas por enzimas de la microflora a las respectivas formas agliconas activas ${ }^{2}$. Cabe mencionar además que el equol, un metabolito de la daidzeina producido en el intestino de solamente $30 \%$ de la población humana occidental, es un potente compuesto bioactivo ${ }^{3}$.

Dependiendo de la variedad de la soya, del lugar geográfico, las condiciones ambientales del cultivo y las características del procedimiento industrial de su elaboración, el contenido total 
de ISO en los extractos de proteína de soya que se comercializan muestran una gran variabilidad (200-800 $\mu \mathrm{g} / \mathrm{g})^{4}$. En Asia el consumo de alimentos derivados de poroto de soya es 20-80 gramos por día, en cambio en occidente el consumo es de 1-5 gramos por día. En humanos los niveles detectados de ISO séricos son directamente dependientes del consumo, ya sea de alimentos ricos en soya o de la ingesta de las ISO como suplemento alimenticio. El nivel promedio de ISO que se detecta en el suero en la población de Japón es de alrededor de 270 $\mu \mathrm{g} / \mathrm{L}$, en cambio en los países occidentales, incluido Chile, la concentración es aproximadamente de $16 \mu \mathrm{g} / \mathrm{L}^{5,6}$.

La similitud estructural que existe entre las ISO de la soya y el $17 \beta$-estradiol les permite unirse a nivel celular con los receptores para estrógenos (ERs). Los ERs son factores transcripcionales inducibles por el ligando. La unión de estradiol y de otros compuestos estrogénicos tales como las isoflavonas estimulan la dimerización del receptor y su asociación con los elementos de respuesta, en la región promotora del ADN, controlando la transcripción de los genes blancos de los ERs ${ }^{7}$. Los ERs existen en dos formas, $\mathrm{ER}_{\alpha} \mathrm{yER} \mathrm{ER}_{\beta}$, y las isoflavonas se unen con mayor afinidad por el $\mathrm{ER}_{\beta}{ }^{7}$. Las ISO poseen un amplio rango de mecanismos de acción ya que sus propiedades biológicas se extienden más allá de su capacidad de unirse a los receptores ERs, incluyendo propiedades antioxidantes ${ }^{8}$, regulación de actividad de algunas enzimas ${ }^{9}$, inhibición de señales celulares ${ }^{10}$ y regulación de la proliferación celular ${ }^{11}$.

Los estudios epidemiológicos y de observación se han focalizado principalmente en los posibles efectos del consumo de alimentos ricos en soya, o de la administración de las ISO como suplemento alimenticio en la prevención de cáncer, enfermedades cardiovasculares y patologías derivadas de alteraciones del sistema endocrino ${ }^{12}$. En esta revisión se analizará el cáncer mamario y los riesgos asociados a esta patología, por alteraciones en la sincronización de la pubertad.

\section{Cáncer mamario}

Los estrógenos son esenciales para el normal desarrollo de la glándula mamaria, regulando tanto la proliferación de las células del epitelio como también el desarrollo de un epitelio saludable. Si bien los niveles fisiológicos de estrógenos juegan un papel fundamental en el normal funcionamiento de la glándula mamaria, la exposición prolongada a altos niveles de los mismos se cree asociada con incrementos en la incidencia de cáncer mamario ${ }^{13}$. Alteraciones de la exposición normal a estrógenos relacionados con pubertad precoz, retardo en la menopausia y terapia de reposición hormonal han sido consideradas como factores de riesgo, mientras que la maternidad a edad temprana, nutrición materna prolongada y retardo en la pubertad son factores que se asocian con menor incidencia de cáncer mamario ${ }^{14}$. Ha sido difícil determinar si las ISO protegen o estimulan el cáncer mamario. Considerando que las ISO pueden tener efectos tanto estrogénicos como antiestrogénicos, se ha sugerido que estos compuestos pueden modular el riesgo de cáncer mamario, siendo estos efectos más importantes si el consumo ocurre antes de la pubertad ${ }^{15}$.

En animales de experimentación las ISO pueden promover o reprimir el crecimiento de tumores. Estudios en ratas establecen que el momento y la duración de la exposición a las ISO son cruciales para la acción. La incidencia de tumores inducidos por agentes químicos disminuye significativamente cuando ratas pre-púberes son tratadas con extractos de poroto de soya o de genisteina ${ }^{16}$. Sin embargo, en ratones, los resultados son menos claros, ya que la exposición a genisteina u otras isoflavonas reduce, estimula o no tiene efectos en la incidencia de tumores mamarios y/o en la multiplicidad y tamaño de los mismos ${ }^{16}$.

¿Cómo explicar la heterogeneidad de los resultados? En los estudios de intervención no hay una comparación entre las acciones de las ISO con los biomarcadores de cáncer a la mama, los niveles locales de estrógenos, como tampoco con los niveles de expresión de ER $\alpha$ y ER $\beta$. Se ha postulado que los efectos de las ISO pueden variar desde un comportamiento anti-estrogénico a una acción estrógenica débil, dependiendo si la mujer tiene bajos o altos los niveles endógenos de estradiol (en mujeres premenopáusicas los valores van de 50 a $400 \mathrm{pg} / \mathrm{ml}$, en tanto que en postmenopáusicas oscilan entre 10 a $20 \mathrm{pg} / \mathrm{ml}$ ). Estudios con animales transgénicos, donde la respuesta a los estrógenos esta acoplada a elementos que inducen el gen de la luciferasa, han permitido aclarar algunas interrogantes. Cuando ratas ovarectomizadas, para evitar la producción endógena de estradiol, fueron tra- 
tadas con ISO la actividad de la enzima luciferasa aumentaba en el hígado, la hipófisis y la glándula mamaria, indicando que la ISO genera una repuesta estrogénica. Por el contrario, cuando las ratas fueron inyectadas con estrógenos, la ingesta de ISO atenuó la acción estimuladora del estradiol sobre la expresión de la luciferasa, es decir, produjo un efecto antiestrogénico ${ }^{17}$. Un reciente meta-análisis de 47 protocolos sugiere que el consumo de soya o de isoflavonas, en mujeres pre o postmenopáusicas, no afecta los niveles circulantes de estrógenos, pero reduce significativamente los niveles de FSH y LH en mujeres premenopáusicas ${ }^{18}$.

Otro factor interesante a considerar es la relación $\mathrm{ER}_{\alpha} \mathrm{y} \mathrm{ER}_{\beta}$ presente en los tejidos blancos. Aunque la capacidad transcripcional de ambos receptores puede ser activada por los estrógenos o por las ISO, la relevancia fisiológica de la regulación transcripcional mediada por el $\mathrm{ER}_{\beta}$ es diferente al de $\mathrm{ER}_{\alpha}$. Por ejemplo, mientras las señales a través de $\mathrm{ER}_{\alpha}$ son mitogénicas e importantes en el desarrollo del tejido mamario femenino, el $\mathrm{ER}_{\beta}$ no promueve la proliferación y carece de efectos estimuladores en el desarrollo del tejido mamario e incluso puede contrarrestar los efectos mediados por $\mathrm{ER}_{\alpha}^{19,20}$. De esta manera, dependiendo del contexto de los receptores de estrógenos en los tejidos blancos, las ISO pueden inducir o reducir la proliferación, situación que no ha sido foco de investigación en ningún estudio de intervención con ISO de soya.

Los estudios a nivel génico han aportado importantes resultados que ayudan a comprender la acción de las ISO en la prevención del desarrollo de tumores. En células en cultivo se ha podido regular la relación entre niveles de $\mathrm{ER}_{\alpha} \mathrm{y} \mathrm{ER}_{\beta}$. En un reciente trabajo, en células de cáncer mamario T47D, que expresan mayoritariamente el $\mathrm{ER}_{\alpha}$, la genisteina, a través de la remodelación del citoesqueleto, induce señales transcriptómicas (producción de RNA) y proteómicas (producción de proteínas) que desencadenan un rápido crecimiento y migración celular ${ }^{21}$. Sin embargo, cuando se induce experimentalmente la expresión tanto de $\mathrm{ER}_{\alpha}$ como de $\mathrm{ER}_{\beta}$, los efectos de genisteina en el crecimiento celular fueron inhibidos, en tanto que se inducen factores que detienen el ciclo celular y otros que inducen apoptosis. De igual manera, en estudios en líneas celulares que contenían o no el $\mathrm{ER}_{\alpha}$, el tratamiento con genisteina o daidzeina afectó en forma diferencial la expresión de varios genes ${ }^{22}$.
Recientes estudios revelan que tanto el tejido mamario normal como el tumoral poseen células troncales mamarias $(\mathrm{CTM})^{23}$. Estas células se caracterizan tanto por su capacidad de autorenovación como también por expresar genes propios de células troncales de origen embrionario. Sorprendentemente, los estrógenos reducen la auto-replicación de las CTM y la expresión de algunos de sus genes, estimulando su diferenciación y promoviendo simultáneamente la proliferación de aquellas células progenitoras más diferenciadas. Dado que el aumento en el número de las células troncales o progenitoras estaría asociado a un incremento en el riesgo de cáncer mamario, los estrógenos paradojalmente tendrían la acción dual al promover, por un lado, la aparición de tumores y por otro lado, reprimir sus funciones. Este importante hallazgo impulsa a definir los efectos de las ISO en las células troncales de la glándula mamaria en diferentes etapas de la vida para establecer ventanas de tiempo en el cuál las ISO pueden tener efectos positivos o negativos.

Interesantemente, el Estudio de la Salud de la Mujer en Shanghai ${ }^{24}$ correlacionó el consumo de isoflavonas de soya en el desarrollo de cáncer mamario, detectando que quienes consumieron mayor cantidad de soya tenían reducido significativamente el riesgo de cáncer mamario. El efecto fue observado solamente en mujeres premenopáusicas, no observándose correlación en mujeres postmenopáusicas.

\section{Pubertad}

Los determinantes de la sincronización de la pubertad no están enteramente establecidos, aunque el inicio de la pubertad en las niñas está asociado con la grasa corporal, la genética y factores medioambientales. Ya que la sincronización de la pubertad podría ser un factor de riesgo para algunos cánceres asociados a hormonas, incluyendo la mama, la próstata y el cáncer testicular, ha surgido recientemente un gran interés por estudiar los efectos de los fitoestrógenos en su desarrollo. En un estudio con niñas sanas de 9 años de edad, se observó que un retardo en el desarrollo mamario va asociado a una ingesta de $2 \mathrm{mg} /$ día de ISO ${ }^{25}$. Aun mayor impacto ha sido provocado por un muy reciente estudio en que se observó que niñas, con ingesta de $1,2 \mathrm{mg} /$ día de ISO a partir de los 2 años 
de edad, experimentaron un retraso de alrededor de 7 meses tanto en el desarrollo mamario (estadio 2 de Tanner) como en la velocidad en el rango de mayor crecimiento de la estatura ${ }^{26}$. En varones de similar edad, la ingesta de 1,4 mg/día de ISO en la dieta no afectó la sincronización de la pubertad. El dimorfismo sexual en algunos marcadores de la pubertad observado con el consumo de ISO puede ser un reflejo del hecho que los estrógenos, referente a los andrógenos, son menos relevantes para el desarrollo genital y esquelético en los varones que en las mujeres. El efecto anti-estrógenico de las ISO observado en las niñas puede ser una consecuencia de su acción inhibitoria a nivel en la actividad de las enzimas aromatasa y $17 \beta$-hidroxiesteroide deshidrogenasa (17 $\beta-H S D)^{9,27}$. La aromatasa es la enzima limitante en los procesos de conversión de androstenediona y testosterona a estrona y estradiol, respectivamente. En este contexto ha sido observada una amplificación en los efectos inhibitorios de mezclas de ISO (como las presentes en las dietas ricas en ISO de la soya), en comparación con la administración de ISO aisladas ${ }^{28}$. La otra enzima que potencialmente puede ser inhibida por las ISO, la 17 $\beta$-HSD, cataliza la interconversión de los relativamente inactivos $17 \beta$-cetoesteroides, estrona y androstenediona, a los activos esteroides sexuales $17 \beta$-hidroxilados, $17 \beta$-estradiol y testosterona, respectivamente.

Como se indico en la sección anterior las ISO pueden también directamente actuar con los $\mathrm{ER}_{\alpha}$ y $\mathrm{ER}_{\beta}$, los cuales tienen acciones estrogénicas regulatorias opuestas.

La menarquía temprana constituye un factor de riesgo para el cáncer de mama y se especula que se podría atribuir a un adelantamiento en la acumulación prolongada de estrógenos. Una ingesta rica en fitoestrógenos del tipo ISO puede generar un retardo en la sincronización de la pubertad en niñas, pero no en niños. Lo anterior puede provocar un entusiasmo generalizado por los potenciales beneficios en la salud que tienen los alimentos derivados de la soya con un alto contenido de ISO. Sin embargo, seriamos negligentes si no consideramos los riesgos que pueden tener las ISO debido a sus propiedades estrogénicas. La capacidad de las ISO para actuar como agonistas/ antagonistas ha llevado a que algunos autores las consideren disruptores endocrinos. La evaluación de la seguridad que representa el consumo de las ISO ha sido comprendida por diversas agencias gubernamentales y no gubernamentales de Europa, Estados Unidos de Norteamérica y Japón. Los estudios tanto epidemiológicos como clínicos en humanos apoyan el planteamiento que las ISO son seguras ${ }^{29}$. Algunos trabajos, en animales de experimentación y con células en cultivo, sin embargo, indican que los productos que contienen ISO podrían representar un peligro para las mujeres con alto riesgo de desarrollar cáncer de mama. Algo similar ocurriría con pacientes con cáncer de mama del tipo receptor de estrógenos positivo que se encuentran bajo tratamiento con moduladores selectivos del receptor de estrógenos (SERM; tamoxifeno y raloxifeno), ya que se ha visto en experimentos con células tumorales mamarias en cultivo que la genisteina elimina la acción antagonista de tamoxifeno sobre el $\mathrm{ER}_{\alpha}{ }^{30}$. Las evidencias acumuladas estimulan la ingesta de alimentos ricos en isoflavonas; sin embargo, para el tipo de mujeres mencionadas anteriormente no es recomendable el consumo (dosis mayores a 20 $\mathrm{mg} /$ día) de suplementos alimenticios con extractos de isoflavonas.

Al momento de escribir este manuscrito, la comunidad científica y médica espera el informe que esta elaborando la Agencia Europea de Seguridad Alimentaria sobre la seguridad del consumo de la soya contrastando los fundamentos científicos de los pros y el contra de las isoflavonas.

\section{Referencias}

1. Messina MJ. Legumes and soybeans: overview of their nutritional profiles and health effects. Am J Clin Nutr 1999; 70: 439-50.

2. Setchell KD, Brzezinski A, Brown NM, Desai PB, Melhem M, Meredith T, et al. Pharmacokinetics of a slowrelease formulation of soybean isoflavones in healthy postmenopausal women. Agric Food Chem 2005; 53 : 1938-44.

3. Setchell KD, Clereci C. Equol: Pharmacokinetics and Biological Actions J Nutr 2010; 140: 1363S-8S.

4. Brown N, Setchell KDR. Animal models impacted by phytoestrogens in commercial chow: implications for pathways influenced by hormones. Lab Invest 2001; 81: 735-47.

5. Garrido A, De la Maza MP, Hirsch S, Valladares L. Soy isoflavones affect platelet thromboxane A2 receptor density but not plasma lipids in menopausal women. Maturitas 2006; 54: 270-6. 
6. Morton MS, Arisaka O, Miyake N, Liam D. Morgan LD, Bronwen A. J, et al. Phytoestrogen Concentrations in Serum from Japanese Men and Women over Forty Years of Age. J Nutr 2002; 132: 3168-71.

7. Kuiper GG, Carlsson B, Grandien K, Enmark E, Haggblad J, Nilsson S, et al. Comparison of the ligand binding specificity and transcript tissue distribution of estrogen receptors alpha and beta. Neuroendocrinology 1997; 138: 863-70.

8. Sarkar FH, Li Y, Wang Z, Kong D. Cellular signaling perturbation by natural products. Cell Signal 2009; 21: 1541-7.

9. Adlercreutz H, Bannwart C, Wähälä K, Mäkelä T, Brunow $\mathrm{G}$, Hase T, et al. Inhibition of human aromatase by mammalian lignans and isoflavonoid phytoestrogens. J Steroid Biochem Mol Biol 1993; 44: 147-53.

10. Muñoz Y, Garrido A, Valladares L. Equol is more active than soy isoflavone itself to compete for binding to thromboxane A (2) receptor in human platelets. Thromb Res 2009; 123: 740-4.

11. Sakamoto T, Horiguchi H, Oguma E, Kayama F. Effects of diverse dietary phytoestrogens on cell growth, cell cycle and apoptosis in estrogen-receptor-positive breast cancer cells. J Neurosci Res 2010; 88: 877-86.

12. Adlercreutz H. Epidemiology of phytoestrogens. Bailieres Clin Endocrinol Metab 1998; 12: 605-23.

13. Eliassen AH, Hankinson SE. Endogenous hormone levels and risk of breast, endometrial and ovarian cancers: prospective studies. Adv Exp Med Biol 2008; 630: 14865.

14. Clemons M, Gross P. Estrogens and the risk of breast cancer. N Engl J Med 2001; 344: 276-85.

15. Adlercreutz H. Phytoestrogen and breast cancer. J Steroid Biochem Mol Biol 2002; 83: 113-8.

16. Warri A, Saarinen NM, Makela S, Hilakivi-Clarke L. The role of early life genistein exposures in modifying breast cancer risk. Br J Cancer 2008; 98: 85-3.

17. Penttinen-Damdimopoulou PE, Power KA, Hurmerinta TT, Nurmi T, van der Saag PT, Mäkela SI. Dietary sources of lignans and isoflavones modulate responses to estradiol in estrogen reporter mice. Mol Nutr Food Res 2009; 53: 996-06.

18. Hooper L, Ryder JJ, Kurzer MS, Lampe JW, Messina MJ, Phipps WR, et al. Effects of soy protein and isoflavones on circulating hormone concentrations in pre- and post-menopausal women: a systematic review and metaanalysis. Hum Reprod Update 2009; 15: 423-40.

19. Bardin A, Boulle N, Lazennec G, Vignon F, Pujol G. Loss of ER \{beta\} expression as a common step in estrogendependent tumor progression. Endocr Relat Cancer 2004; 11: 537-51.

20. Ström A, Hartman J, Foster JS, Kietz S, Wimalasena J, Gustafsson JA. Estrogen receptor $\beta$ inhibits $17 \beta$-estradiol-stimulated proliferation of the breast cancer cell line T47D. Proc Natl Acad Sci U.S.A. 2004; 101: 1566-71.

21. Sotoca AM, Gelpke MD, Boeren S, Ström A, Gustafsson JÅ, Murk AJ, et al. Quantitative proteomics and transcriptomics addressing the estrogen receptor subtypemediated effects in T47D breast cancer cells exposed to the phytoestrogen genistein. Mol Cell Proteomics 2010, doi: 10.1074/mcp.M110.002170.

22. Satih S, Chalabi N, Rabiau N, Bosviel R, Fontana L, Bignon YJ, et al. Gene expression profiling of breast cancer cell lines in response to soy isoflavones using a pangenomic microarray approach. OMICS 2010; 14: 231-38.

23. Simöes BM, Piva M, Iriondo O, Comcills V, López-Ruiz JA, Zabalza I, et al. Effect of estrogen on the proportion of stem cells in the breast. Breast Cancer Res Treat 10.1007/s10549-010-1169-4.

24. Lee SA, Shu XO, Li H, Yang G, Cai H, Wen W, et al. Adolescent and adult soy food intake and breast cancer risk: results from the Shanghai Women's Health Study. Am J Clin Nutr 2009; 89: 1920-6.

25. Wolff MS, Britton JA, Boguski L, Hochman S, Maloney $\mathrm{N}$, Serra N, et al. Environmental exposures and puberty in inner-city girls. Environ Res 2008; 107: 393-400.

26. Cheng G, Remer T, Prinz-Langenohl R, Blaszkewicz M, Degen GH, Buyken AE. Relation of isoflavones and fiber intake in childhood to the timing of puberty Am J Nutr 2010; 92: 556-64.

27. Brooks JD, Thompson LU. Mammalian lignans and genistein decrease the activities of aromatase and 17betahydroxysteroid dehydrogenase in MCF-7 cells. J Steroid Biochem Mol Biol 2005; 94: 461-7.

28. Rice S, Mason HD, Whitehead SA. Phytoestrogens and their low dose combinations inhibit mRNA expression and activity of aromatase in human granulosa-luteal cells. J Steroid Biochem Mol Biol 2006; 1012: 16-25.

29. Munro IC, Harwood M, Hlywka JJ, Stephen AM, Doull J, Flamm WG, et al. Soy isoflavones: a safety review. Nutr Rev 2003; 61: 1-33.

30. Helferich WG, Andrade JE, Hoagland MS. Phytoestrogens and breast cancer: a complex story. Inflammopharmacology 2008; 16: 219-26. 